\section{Die Chance für nachhaltige (Unternehmens-)Entwicklung?}

\author{
Die Einbindung von NutzerInnen in die Produktentwicklung kann dazu beitra- \\ gen, dass sowohl auf Nutzer- als auch auf Unternehmensseite Lernprozesse in \\ Richtung Nachhaltigkeit stattfinden. So lautet zumindest das Credo partizipa- \\ tiver Produktentwicklung. Ob die Integration des Alltagswissens der Nutzer- \\ Innen über den Gebrauch eines Produkts in die Produktenwicklung ein klimaf- \\ reundliches Produkt entstehen lässt, wird momentan untersucht.
}

$\mathrm{D}$ Von Maria Hage und Esther Hoffmann ie Agenda 21 sieht die nicht nachhaltigen Verbrauchs- und Produktionsmuster, insbesondere in den Industrieländern, als Hauptursache für die allmähliche Zerstörung der globalen Umwelt. Hierbei kommt Unternehmen eine besondere Verantwortung zu, da das Angebot umweltfreundlicher Produkte Voraussetzung für umweltgerechtes Verbraucherverhalten ist. Aber auch VerbraucherInnen tragen Verantwortung, der sie zurzeit vor allem durch ihre Kaufentscheidung Rechnung tragen. Ihre direkte Einflussnahme auf die Produktgestaltung ist gering.

Die ökologischen Folgewirkungen von Produkten werden zum großen Teil durch die Produktgestaltung festgelegt, weshalb die Umweltverträglichkeit von Produkten bereits bei ihrer Entwicklung zu berücksichtigen ist. Hierzu sollten frühzeitig alle Phasen des Produktlebenszyklus betrachtet werden. Für die Hersteller sind die Nutzungsphase und die dort vorhandenen ökologischen Optimierungspotenziale häufig ein blinder Fleck, da sie über das Nutzerverhalten im Umgang mit Produkten nur unvollständige Annahmen treffen können.

\section{Austausch findet selten statt}

NutzerInnen stellen zwar zentrale Stakeholder der Unternehmen dar, ihre Interessen werden aber häufig nur mittelbar über Marketing und Marktforschung in die Produktentwicklung einbezogen. Dabei setzen Marketing und Marktforschung oft erst bei relativ weit fortgeschrittenen Produktkonzepten, bestehenden Produkten oder durch Trendforschung bei allgemeinen gesellschaftlichen Trends an. Ein dialogischer Austausch, durch den nicht nur die Wünsche, sondern auch das Handlungs- und Alltagswissen der KonsumentInnen genutzt werden, ist selten. Dies gilt auch für den Rückfluss von Informationen an die KonsumentInnen. Dieser wäre jedoch wichtig, um Lernprozesse anzuregen und Verhaltensänderungen auf beiden Seiten auszulösen.

Dem Projekt Gesellschaftliches Lernen und Nachhaltigkeit (GELENA) liegt die Annahme zugrunde, dass Nutzerinnen und Nutzer über ein spezifisches Alltagswissen verfügen, welches zur Weiter- und Neuentwicklung eines Produktes beitragen kann (1). Das Wissen über den alltäglichen Umgang mit einem Produkt oder einer Dienstleistung kann bei frühzeitiger Integration in die Neugestaltung eines Produktes dazu beitragen, den Energie- und Ressourcenverbrauch in der Nutzungsphase zu verringern. Auch in der neueren Innovationsforschung wird KundInnen eine kritische Rolle in Innovationsprozessen zugesprochen.

Die Zusammenführung des technischen Fachwissens der Produktentwickler mit dem alltagspraktischen Erfahrungswissen der NutzerInnen kann $\mathrm{zu}$ wechselseitigen Lernprozessen führen. Die Lernprozesse können bei den KonsumentInnen zum empowerment beitragen, indem ihnen zum einen mehr Gestaltungsspielraum eingeräumt wird und sie zum anderen durch einen bewussten Umgang mit Produkten ihre Handlungsspielräume im Alltag erweitern. Hierzu sollten KonsumentInnen an Ideenentwicklungs-, Bewertungs- und Selektionsprozessen in der Produktentwicklung beteiligt werden. Der Einbezug von KonsumentInnen in die Produktentwicklung setzt auf Seiten der Unternehmen die Bereitschaft zu Lern- und Veränderungsprozessen voraus, die sowohl ihre Einstellung zu KonsumentInnen als auch die bisherige Organisation von Produktentwicklungsprozessen umfassen.

So stellt sich zumindest die Theorie dar. In der Praxis zeigen sich allerdings eine Reihe von hemmenden Faktoren.

\section{Hemmende Faktoren}

Hemmnisse für die Beteiligung von NutzerInnen an der Produktentwicklung bestehen im Fehlen geeigneter Methoden, in der Ausdifferenzierung der Konsumentenanforderungen infolge der Pluralisierung von Lebensstilen, in der Unterschiedlichkeit des Wissens zwischen Herstellern und VerbraucherInnen und den damit verbundenen Übersetzungsproblemen und nicht zuletzt in der unterschiedlichen Macht- und Ressourcenverteilung (2). Als problematisch erweist sich auch die Auswahl der NutzerInnen. Wer soll beteiligt werden und nach welchen Kriterien wird ausgewählt? Wie können die beteiligten NutzerInnen ein realistisches Bild der tatsächlichen ProduktnutzerInnen und ihrer Bedürfnisse abgeben? Hinzu kommt ein klassisches Trittbrettfahrerproblem: Diejenigen, die sich engagieren, schaffen Nutzen auch für Unbeteiligte und es stellt die Frage nach der angemessenen Entlohnung (3). NutzerInnen sind zu-

\section{Grafik 1: Modell partizipativer Produktgestaltung}

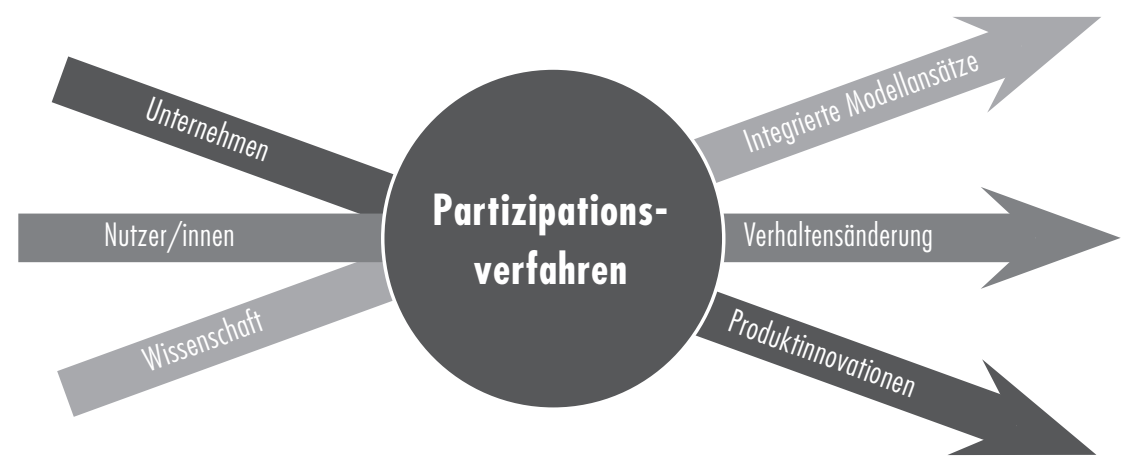


dem häufig an einer Einbindung nicht interessiert, da es für sie einfacher ist, sich mit bestehenden Produkten abzufinden. Weiterhin ist aus einer dynamischen Perspektive zu berücksichtigen, dass sich Nutzerbedürfnisse und Ansprïche verändern und diejenigen aus der Entwicklungsphase nicht notwendigerweise mit denen bei Produktanwendung übereinstimmen müssen.

\section{- Wechselseitige Lernprozesse}

Ziel des GELENA-Projektes ist die Entwicklung und praktische Erprobung eines partizipativen Produktentwicklungsverfahrens. Im Zentrum dieses Verfahrens stehen NutzerInnen und Unternehmen. Anhand vereinfachter Klimamodelle sollen die Auswirkungen der durch den Menschen verursachten Klimaveränderung anschaulich gemacht werden. Dies soll sowohl bei den KonsumentInnen als auch bei den beteiligten UnternehmensvertreterInnen zu Bewusstseinsbildungs- und Lernprozessen führen. Diese Lernprozesse werden durch direkte Interaktion der Unternehmen mit potenziellen KundInnen und den gemeinsamen Dialog über Produktnutzung intensiviert.

Nach van den Ven kann das Zusammentreffen mit KundInnen Unternehmensvertreter motivieren, sich mit Kundenwünschen und sich ändernden Umfeldbedingungen auseinander zu setzen (4). Durch die Einbeziehung mehrerer Unternehmensmitglieder aus verschiedenen Abteilungen können in der Folge innerbetriebliche Lernprozesse auch über die direkt einbezogenen Personen hinaus angestoßen werden.

Während es bereits anerkannte Methoden zur Integration industrieller KundInnen in die Entwicklung von Produkten gibt, liegen wenig Erfahrungen mit der direkten Einbeziehung von privaten NutzerInnen im Allgemeinen und unter Berücksichtigung sozial-ökologischer Ziele vor.

Raabe kommt in einer Untersuchung zu Konsumentenbeteiligung an der Produktentwicklung zu dem Ergebnis, dass es sich in der Praxis eher um einen Dialog zwischen Unternehmen und KonsumentInnen handelt und den KonsumentInnen meist keine wirklichen Mitbestimmungsmöglichkeiten eingeräumt werden (5). Um dem normativen Anspruch an Partizipation gerecht zu werden, wäre jedoch ein gewisses Maß an Mitbestimmungsmöglichkeiten verbunden mit einem Empowerment der KonsumentInnen erforderlich. Formen der aktiven Konsumentenbeteiligung wurden bislang vorwiegend in den Niederlanden entwickelt und eingesetzt. Dort wurde das Konzept des Konstruktive Technologie Bewertung aus Konsu- mentensicht (cCTA) entwickelt und in verschiedenen Produktbereichen erprobt. So wurden zum Beispiel im Projekt „Zukunftsbilder von Konsumenten" in mehreren aufeinander aufbauenden Workshops am Beispiel von neuen Eiweißnahrungsmitteln Zukunftsvisionen aus Konsumentensicht entwickelt (6). Hierbei waren Stakeholder aus verschiedenen gesellschaftlichen und wirtschaftlichen Bereichen eingebunden und es kam nicht zu einem direkten Zusammentreffen von NutzerInnen mit einem entwickelnden Unternehmen. Im Gegensatz dazu operiert der Script-Ansatz vor einem techniksoziologischen Hintergrund. Hier sollen unter Beteiligung von KonsumentInnen verhaltenssteuernde Elemente eines Produktes in Richtung energiesparendes Verhalten umkonstruiert werden, wobei die KonsumentInnen mehr beforscht und beobachtet und weniger aktiv einbezogen werden (7). Diese und ähnliche Ansätze und Methoden sollen im Rahmen des GELENA-Projektes ausgewertet und weiter entwickelt werden.

Um nachhaltigkeitsorientierte Lernprozesse anzuregen baut GELENA bei der Veranschaulichung der Klimaeffekte auf Erfahrungen aus dem Participatory Integrated Assessment (PIA) auf. Ein Beispiel ist das europäische ULYSSES-Projekt, in dem die Einbindung von computergestützten Simulationen in Dialogprozesse von Laien über Klimaänderungen, deren Ursachen und mögliche Gegenstrategien untersucht wurde (8).

\section{- Workshops geplant}

Das Projekt befindet sich derzeit in einer Sondierungs- und Konzeptionsphase. Im Jahr 2005 soll das partizipative Produktentwicklungsverfahren in die Praxis umgesetzt werden. Welche Produkte und Dienstleistungen für ein solches Verfahren geeignet sind, wird zur Zeit in den Bedürfnisfeldern Bauen und Wohnen, Mobilität sowie Information und Kommunikation untersucht (9). Die bisherige Planung für das Verfahren sieht drei aufeinander aufbauende Workshops vor, an denen jeweils UnternehmensvertreterInnen aus verschiedenen Unternehmensbereichen und NutzerInnen teilnehmen. Die NutzerInnen werden über Anzeigen geworben und nach bestimmten Kriterien wie Familiensituation und Zeitbudgets ausgesucht. In den Workshops wird durch vereinfachte Klimamodelle der Zusammenhang von Kohlendioxid-Emissionen und Klimaänderung veranschaulicht. Mit einem Kohlendioxid-Bilanz-Modell können die TeilnehmerInnen die Emissionen bei verschiedenen Produktvarianten selbst berechnen. Die Workshops sollen im Zeitraum von einem halben bis einem Jahr stattfinden, um ausreichend Zeit zwischen den Sitzungen zu lassen. Dies ist zum einem wichtig, damit die erarbeiteten Ergebnisse im Unternehmen rückgekoppelt werden können und Weiterentwicklungen von Seiten des Unternehmens in das Verfahren gespeist werden können. Zudem ist es von Bedeutung, um den teilnehmenden NutzerInnen genug Zeit für Reflektions- und Diskussionsprozesse zu lassen. Das Verfahren wird durch eine Begleitforschung evaluiert, um zu ermitteln, inwieweit die erwarteten Lernprozesse tatsächlich stattfinden.

\section{Anmerkungen}

(1) GELENA ist eine wissenschaftliche Nachwuchsgruppe im Rahmen des Förderschwerpunktes Sozial-ökologische Forschung des Bundesministeriums für Bildung und Forschung. Das Projekt wird vom Institut für ökologische Wirtschaftsforschung (IÖW) in Kooperation mit der Universität Oldenburg bearbeitet. Nähere Informationen unter www.gelena.net.

(2) Weller, I.: Ökologische Stoff- und Produktinnovation. Analyse der Gestaltungsmacht privater Konsumentinnen und Konsumenten am Beispiel Textilien. In: Weller, I./ Hoffmann, E./ Hofmeister, S. (Hg): Nachhaltigkeit und Feminismus: Neve Perspektiven - Alte Blockaden. Bielefeld 1999.

(3) Hansen, U.: Die Stellung des Konsumenten im Prozess der unternehmerischen Produktentwicklung. In: Marketing ZFP. 2/1982, Heft 1 S. 27-36.

(4) Ven, A. H.: Central Problems in the Management of Innovation. In: Management Science 32(5) 1986, S. 590-607.

(5) Raabe, T.: Konsumentenbeteiligung an der Produktinnovation. Frankfurt 1993.

(6) Fonk, G./ Hamstra, A: Toekomstbeelden van Consumenten voor Novel Protein Foods. Illustratieproces voor consumenten van Novel Protein Foods. DTO, DT0 werkdocument VN 12. Delft 1996.

(7) Jelsma, J.: Design of Behaviour Steering Technology. Summer academy of technology studies. Graz 2000.

(8) Vgl. etwa ZIT (Hg.): Ulysses. Urban Lifestyles, Sustainability and Integrated Environmental Assessment. Final Report. Darmstadt 1999.

(9) Die Ergebnisse der Bedürfnisfeldstudien sind voraussichtlich ab April 2004 unter www.gelena.net abzurufen.

\section{Die Autorinnen}

Maria Hage und Esther Hoffmann sind wissenschaftliche Mitarbeiterinnen im Forschungsfeld Ökologische Unternehmenspolitik am Institut für ökologische Wirtschaftsforschung (IÖW).

Kontakt: IÖW, Potsdamer Str. 105, 10785 Berlin. Tel. 030-8845940, Fax 030-8825439, E-Mail: maria.hage@ioew.de; esther.hoffmann@ioew.de 
(c) 20I0 Authors; licensee IÖW and oekom verlag. This is an article distributed under the terms of the Creative Commons Attribution Non-Commercial No Derivates License (http://creativecommons.org/licenses/by-nc-nd/3.o/), which permits unrestricted use, distribution, and reproduction in any medium, provided the original work is properly cited. 